\title{
Dificultades de aprendizaje en escolares no asociados a la discapacidad
}

\author{
Learning difficulties at school not associated with disability \\ Dificuldades de aprendizagem em estudantes não associados à discapacidade
}

DOI: http://dx.doi.org/10.21803\%2Fpenamer.11.21.532

Rosa Esperanza Chabla Sarabia https://orcid.org/0000-0001-9667-8710

María Isabel Alvarez Lozano https://orcid.org/0000-0001-8029-1933

\section{¿Cómo citar este artículo?}

Chabla, R. \& Alvarez, M. (2018). Dificultades de aprendizaje en escolares no asociados a la discapacidad. Pensamiento Americano, 11(21), 169-180 DOI:http://dx.doi.org/10.21803\%2Fpenamer.11.21.532

\section{Resumen}

Esta investigación científica-educativa centra su interés en identificar las dificultades de aprendizaje no asociadas a la discapacidad en la Unidad Educativa Fiscomisional La Salle, de la ciudad de Azogues, en niños de sexto y séptimo año de Educación General Básica. Las Dificultades de Aprendizaje tienen varias manifestaciones según el DSM - V, (2013) que parte desde trastornos de tipo perceptivo hasta los problemas específicos del aprendizaje, donde se encuentra la lectura, escritura y matemáticas. El método utilizado fue el descriptivo y cuantitativo, mediante la aplicación del cuestionario de evaluación de Problemas de Aprendizaje (CEPA) a los docentes, se ha logrado determinar que existe el $20 \%$ de estudiantes que presentan dificultad en alguna área específica de aprendizaje, considerando los 55 de la población aplicada, el índice es muy significativo. La importancia de esta investigación es permitir a los docente detectar Dificultades de Aprendizaje no asociados a la discapacidad dentro del aula de clases, con el apoyo de herramientas que sean de uso adecuado, y de esta manera proponer estrategias de solución basadas en la realidad que se está viviendo, ya que en la ciudad de Azogues no existe estudios de este tipo.

PALABRAS CLAVE: Dificultades de Aprendizaje, Estudiantes, Áreas, No asociados a la discapacidad.

\begin{abstract}
This scientific-educational research centers its interest in identifying learning difficulties not associated with disability in the Educational Unit La Salle" la Unidad Educativa Fiscomisional La Salle", in the city of Azogues, in sixth and seventh -year children I Basic General Education. Learning Difficulties have several manifestations according to the DSM - V, which starts from perceptive type disorders to the specific problems of learning, where reading, writing and mathematics are found. The method used was the descriptive and quantitative, by applying the questionnaire of evaluation of Learning Problems (CEPA) to teachers, it has been determined that there is $20 \%$ of students who have difficulty in a specific area of learning, considering the 55 of the applied population, the index is very significant. The importance of this research is to allow teachers to detect Learning Difficulties not associated with disability within the classroom, with the support of tools that are suitable for use, and thus propose solution strategies based on the reality that is being lived, since in the city of Azogues there are no studies of this type.
\end{abstract}

KEYWORDS: Learning Difficulties, Students, Areas, not associated with disability. 


\section{Resumo}

Esta pesquisa científico-educativo centra seu interesse em identificar as dificuldades de aprendizagem não associadas à incapacidade na Unidade Educativa Fiscomisional La Salle, da cidade de Azogues, em crianças de sexto e sétimo ano de Educação Geral Básica. As dificuldades de aprendizagem têm várias manifestações segundo o DSM-V, que parte desde os transtornos de tipo perceptivo até os problemas específicos da aprendizagem, onde se encontra a leitura, a escrita e as matemáticas. $O$ método utilizado foi o descritivo e quantitativo, mediante a aplicação do questionário de avaliação dos Problemas de Aprendizagem (CEPA) aos docentes, se conseguiu determinar que existem $20 \%$ de estudantes que apresentam dificuldade em alguma área específica de aprendizagem, considerando os 55 da população aplicada, o índice é muito significativo. A importância desta pesquisa reside em que permitirá ajudar aos docentes a detectar Dificuldades de Aprendizagem não associados à incapacidade dentro da sala de aula, com o apoio de ferramentas que sejam de uso adequado, e desta maneira propor estratégias de solução baseadas na realidade em que estão vivendo, já que na cidade de Azogues não existem estudos desse tipo.

PALAVRAS CHAVE: Dificuldades de Aprendizagem, Estudantes, Áreas, Não associadas à incapacidade.

\section{Perfil}

Mención Sicología Educativa y Orientación Vocacional. Universidad Católica de Cuenca Sede-Azogues rosy.cha1993@gmail.com.

\section{Rosa Esperanza Chabla} Sarabia

Licenciada en Ciencias de la Educación

María Isabel Alvarez Lozano Licenciada en Ciencias de la Educación

Mgs. Educación Especial

Universidad Católica de Cuenca Sede Azogues

mialvarezl@ucacue.edu.ec 


\section{Introducción}

$\mathrm{D}$ urante la etapa escolar, existen niños que muestran dificultades en las áreas de aprendizaje, si esto no llega a solucionarse podría mostrar problemas para aprender en años superiores. Los docentes de las instituciones educativas desconocen los tipos de dificultades de aprendizaje no asociados a la discapacidad que existen y no saben cómo detectar a tiempo, porque no tienen un instrumento adecuado que los apoye o desconocen de la existencia de aquello.

Llamamos Dificultades de Aprendizaje no asociados a la discapacidad a aquellas que presentan un desorden psicológico básico, en donde se asocia y se involucra falencias en el lenguaje y la compresión, ya sea oral o escrito, a su vez los escolares presentan dificultad para pensar, leer, hablar, escribir y realizar cálculos matemáticos o de razonamiento. Por el contrario, las dificultades asociadas a la discapacidad son las que se encuentran en estrecha relación con fallas o limitaciones, ya sea en la audición, visión, motriz, intelectual, mental, etc. es decir son discapacidades de tipo físicas, perceptivas y cognitivas (Pacheco, 2014).

Las dificultades por aprender podrían verse ligado directamente a un problema de tipo extrínsecos presentando problemas de atención en clases, retrasos en el desarrollo, problemas comportamentales, etc., puesto que, existen estudiantes que en ciertas áreas de estudio la consideran con mayor dificultad y no saben de qué manera resolverlo. El docente no actúa de manera favorable, pero; no es porque no quiera, sino, por el desconocimiento que tiene en detección de las dificultades. Si los docentes supieran detectar dificultades de aprendizaje, dentro del aula desarrollarían espacios de aprendizaje interactivo y permitirá absorber conocimientos casi de manera in- consciente, creando un hábito de enseñanza que se verá reflejado en el aprovechamiento del cuerpo estudiantil.

El conocimiento de las dificultades de aprendizaje no asociados a la discapacidad tiene varias concepciones, y debe de ser abordado dentro de las instituciones educativas y no externamente. Nicasio García acota que:

"Dificultad de Aprendizaje es un término general que se refiere a un grupo heterogéneo de trastornos que se manifiesta por dificultades significativas en la adquisición y el uso de la escucha, habla, lectura, escritura, razonamiento o habilidades matemáticas. Estos trastornos son intrínsecos al individuo, suponiéndose debidos a la disfunción del sistema nervioso central, y pueden ocurrir a lo largo del ciclo vital..........." (García, 2010).

Los estudios realizados por la Universidad de Málaga, consideran que, "La consecuencia escolar de estos problemas es que los alumnos rinden por debajo de su capacidad y que, si son ignorados y no remiten, las consecuencias para el estudiante, y para el sistema educativo, pueden ser muy importantes. Ponen de manifiesto de forma periódica que un numeroso grupo de estudiantes, según la edad, superando el $50 \%$ de la población escolar, donde comete errores importantes en la comprensión de textos sencillos, en la composición escrita, en la realización de fáciles cálculos matemáticos; y que de un 12,4\% al 14\%, tienen problemas para seguir el ritmo normal de clase, a pesar de que no presentan ningún tipo de déficit" (Romero Pérez \& Cerván, 2005).

No existe estudios realizados a nivel nacional sobre este tema, las investigaciones existentes tanto a nivel nacional e internacional se centran en las dificultades específicas de aprendizaje. En todo centro educativo va 
a existir niños con diferentes dificultades de aprendizaje no asociados a la discapacidad. Lo que difiere de aquello es que, en la actualidad ya existen mecanismos que ayudan a detectar y superar, sólo que se debe capacitar a los docentes en la utilización correcta de los instrumentos que están a su alcance.

Para la realización del estudio de detección de dificultades de aprendizaje no asociados a la discapacidad, se utilizó el cuestionario (CEPA), del (Bravo Valdivieso, 1979), que está dirigido a docentes para evaluar cuantitativa y cualitativamente las dificultades que presenta el estudiante en las diferentes áreas de estudio, para que de esta manera pueda emitir un criterio fundamentado en la realidad investigada, saber cuánto afecta en el ámbito educativo y si todo esto puede traer posibles repercusiones en la vida del escolar.

Los resultados son confiables, pues, el cuestionario ha sido utilizado para varias investigaciones, el Dr. Luis Bravo Valdivieso fue el primero en utilizar y comprobar su veracidad. En este trabajo se comprueba la hipótesis plateada, y que dentro del centro educativo investigado de la cuidad de Azogues, aún prevalece la existencia de niños con dificultades de aprendizaje. En el presente trabajo se detalla la información de los resultados obtenidos, donde se tomó como referencia el número total de estudiantes, para la interpretación, análisis y graficación de datos.

\section{Metodología}

Las Dificultades en el Aprendizaje pueden darse a lo largo de la vida, esto se presenta antes de la adolescencia viéndose afectado el proceso educativo de enseñanza - aprendizaje formal e informal. Una Dificultad de Aprendizaje no asociada a la discapacidad interfiere lograr un buen aprendizaje, mismo que no puede realizarse mediante métodos ordinarios, han de introducirse otros métodos especiales de introducción (Fiuza Asorey \& Fernández Fernández, 2014).

Para la investigación se seleccionó a la población total de los estudiantes de sexto y séptimo año de Educación General Básica, que sumaban 55 integrantes, porque es en estos cursos donde más se evidencia las dificultades de aprendizaje que presentan los niños. Junto con los docentes de aula se procedió a evaluar con la ficha a estudiante por estudiante, donde se revisaba cada área y si tenían dificultad en la misma, ya que, el intermediario de aquello era el docente, quien durante todo el lectivo, pudo visualizar que niño o niña, mostraba falencias en su aprendizaje.

Para determinar las Dificultades de Aprendizaje en niños de sexto y séptimo año de Educación General Básica no asociados a la discapacidad, en la Unidad Educativa Fiscomisional La Salle de la ciudad de Azogues, se tomó y se aplicó una ficha de observación elaborado por el Dr. Luis Enrique Valdivieso, dirigido a docentes, para obtener una evaluación cuantitativa y cualitativa de las dificultades que tiene cada estudiante para aprender en comparación con los compañeros de curso, en las áreas de recepción de información, lenguaje oral, atención, concentración, memoria, lectura, escritura, cálculo, aprendizaje e inteligencia, que no tienen relación con la discapacidad.

El bajo rendimiento académico ha sido una condición muy investigada en todos los ámbitos de las disciplinas que lo abordan, con la finalidad tanto de diagnosticarlo como de intervenirlo. El cuestionario de dificultades de aprendizaje (CEPA) es un instrumento adecuado para la evaluación realizada por el profesor en el aula de clase y de gran utilidad en la investigación (Bravo Valdivieso, 1979) citado por (Betancurt, 2010) 
La variable independiente del estudio fue las dificultades de aprendizaje en los estudiantes de sexto y séptimo año de EGB. La variable dependiente fue que no estén asociados a la discapacidad. Variables que ayudaron a la detección de estudiantes que tenían dificultades en su aprendizaje. Los resultados obtenidos se calificaron por áreas, donde se evidenciaba que los estudiantes se encontraban bien en un área específica, pero en otra sí presentaba dificultades. Las áreas evaluadas fueron las siguientes:

1. Recepción de la información: Se encarga de evaluar la habilidad del niño para entender y retener toda la información que brinda el docente.

2. Expresión del lenguaje oral: Evalúa el uso adecuado del lenguaje oral, al comunicarse y exponer tareas.

3. Atención - concentración - memoria: Evalúa la madurez del niño, al concentrarse, poner atención y su memoria en el trabajo escolar.

4. Errores de lectura: Evalúa al niño si se confunde al leer, al decodificar con fluidez y la capacidad de retención.

5. Errores de escritura: Evalúa lo que el niño co- pia y escribe, si su escritura es legible o espontánea, y su ortografía.

6. Matemáticas: Evalúa la capacidad de efectuar y comprender las operaciones básicas y los números ordinales y cardinales.

7. Aprendizaje e Inteligencia: Los docentes evalúan la capacidad de aprender de los niños, dentro del castellano y las matemáticas, y la capacidad intelectual del niño, según su percepción.

\section{Resultados}

Los resultados obtenidos del Cuestionario de Evaluación de Problemas de Aprendizaje (CEPA), aplicada a cada uno de los docentes de 6to y 7mo año de Educación General Básica de la Unidad Educativa Fiscomisional La Salle de la ciudad de Azogues, misma que generó resultados muy importantes para conocer la existencia de las Dificultades de Aprendizaje no asociadas a la discapacidad, en los 55 estudiantes, durante el lectivo 2016 - 2017. Para el estudio a continuación se refleja de manera estadística los datos obtenidos de esta investigación considerando los aspectos de mayor relevancia.

De acuerdo a los datos proporcionados por la institución en el sexto año de Educación Gene-

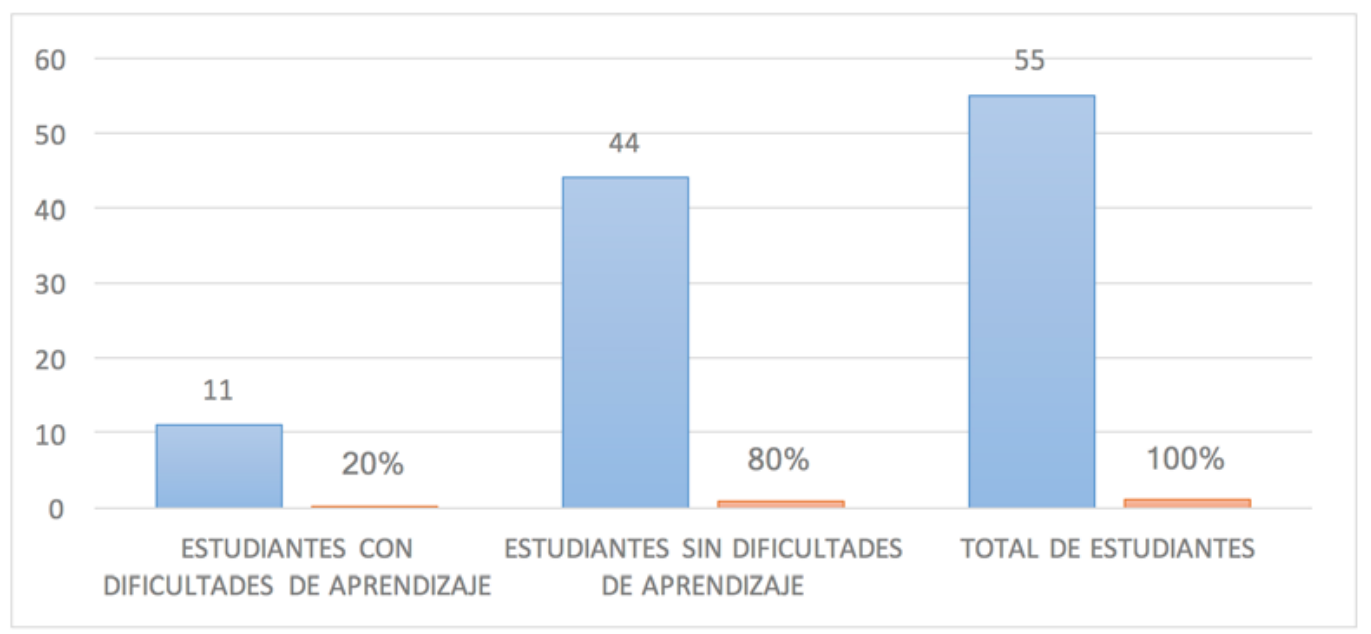

Grafico 1. Total de casos

Fuente: Investigación de campo (Casos de niños y niñas de $6^{\circ}$ y $7^{\circ}$ de EGB con Dificultades de Aprendizaje no asociados a la Discapacidad en la Unidad Educativa Fiscomisional La Salle de la ciudad de Azogues) Elaboración propia. 


\section{ÁREA DE ERRORES DE ESCRITURA}

51

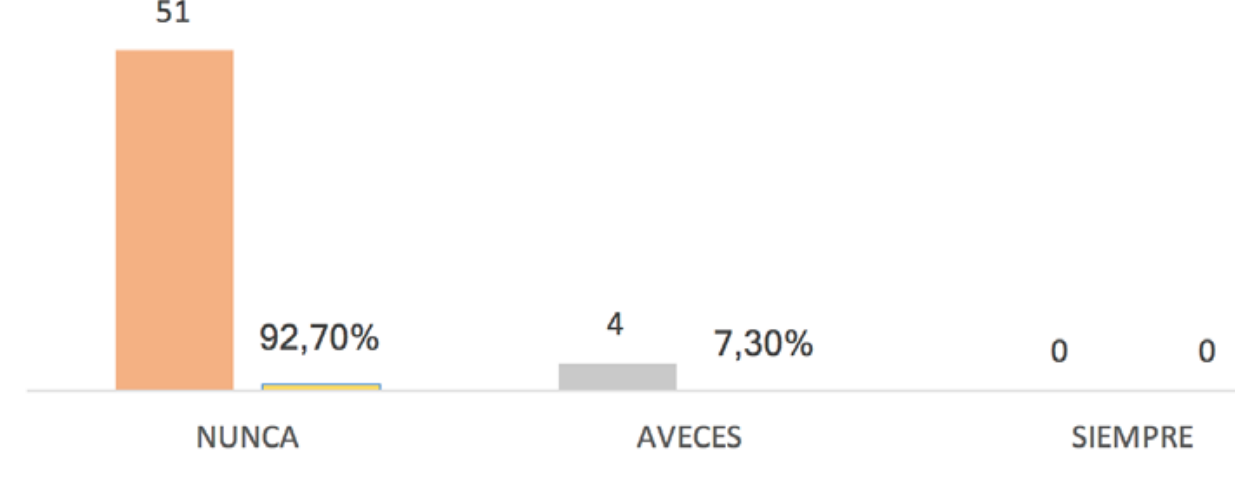

Grafico 2. Area de Errores de escritura

Fuente: Investigación de campo (Casos de niños y niñas de $6^{\circ}$ y $7^{\circ}$ de EGB con Dificultades de Aprendizaje no asociados a la Discapacidad en la Unidad Educativa Fiscomisional La Salle de la ciudad de Azogues) Elaboración propia.

ral Básica existen 30 estudiantes y en el séptimo 25 estudiantes, durante el lectivo 2016 -2017. Los docentes de sexto y séptimo, reportan 11 casos de estudiantes con Dificultades de Aprendizaje no asociados a la Discapacidad, mismo que corresponde al $20 \%$, y es preocupante debido a la cantidad de población aplicada, a esta población resultante los docentes han generado observaciones durante el lectivo, en los casos detectados, ha evidenciado en los estudiantes un rendimiento académico bajo y dificultades en el desarrollo de las tareas en clase. En el centro educativo, el 92,7\% de los estudiantes observa- dos nunca han presentado errores en la escritura, mientras que el $7,3 \%$ a veces muestra dificultad al momento de realizar copias, no tienen legibilidad en su escritura, se les dificulta escribir un dictado, presentan fallas ortográficas y una escritura espontánea. Los docentes alegan que estas fallas se deben a que los niños no han sido estimulados correctamente en la escritura en los primeros años de escolarización.

Cabe recalcar que, de las áreas evaluadas, los estudiantes en mayor número, presentan una escritura correcta, donde los docentes no ven

\section{ÁREA DE MATEMÁTICAS}

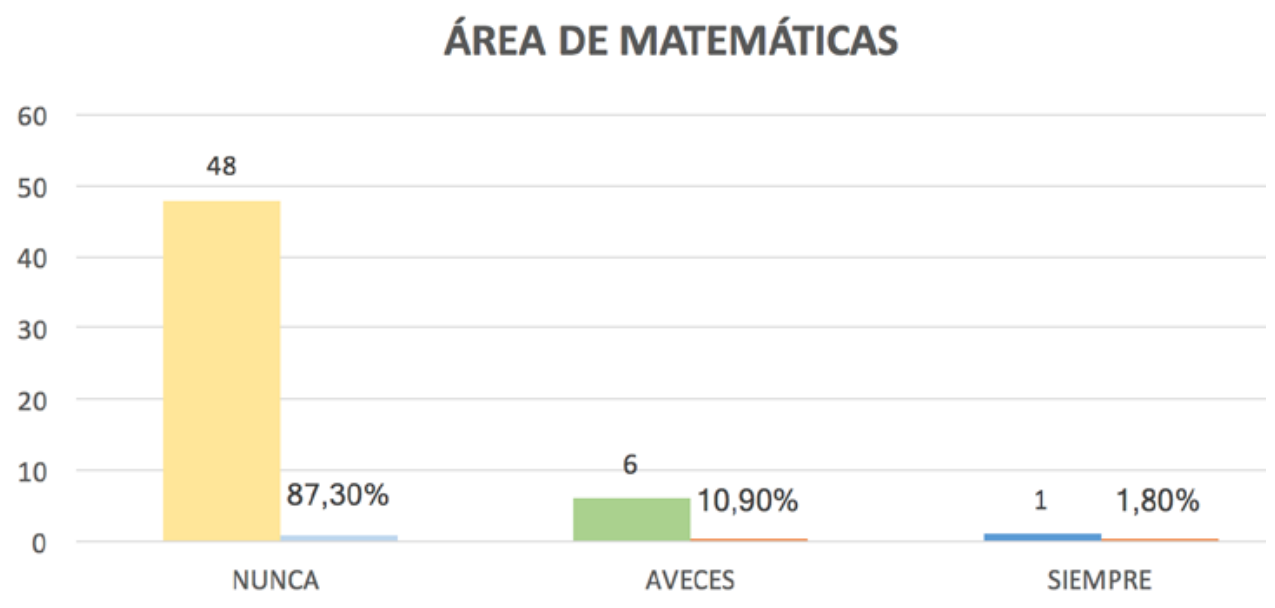

Grafico 3. Área de Matemáticas

Fuente: Investigación de campo (Casos de niños y niñas de $6^{\circ}$ y $7^{\circ}$ de EGB con Dificultades de Aprendizaje no asociados a la Discapacidad en la Unidad Educativa Fiscomisional La Salle de la ciudad de Azogues) Elaboración propia 
dificultad alguna o un retroceso en su aprendizaje, pero; les preocupa aquellos estudiantes que fallan en la escritura, porque temen que esto afecte en el futuro si no es corregido a tiempo. En la Figura 2 se pudo evidenciar el resultado en el área de escritura, o conocido como Dislexia según el DSMV, en la unificación de criterios.

Con el cuestionario de Evaluación de Problemas de Aprendizaje, aplicada por cada uno de los docentes, se puede corroborar que, en el área de matemáticas los estudiantes presentan mayor dificultad, pues muestra un porcentaje alto entre las demás áreas, ya que, el $10,9 \%$ a veces ha tenido dificultad y el 1,8\% siempre muestra problemas. Los niños y niñas según manifiestan los docentes, tienen mayor dificultad en las operaciones básicas, como la suma, resta, multiplicación y división, ya que, en los primeros años de escolarización no fueron reforzados correctamente, por ello su capacidad para efectuar las operaciones aritméticas y comprender su significado es bajo.

\section{Discusión}

Para identificar las dificultades de aprendizaje no asociadas a la discapacidad en los estudiantes de sexto y séptimo año de Educación General Básica de la Unidad Educativa Fiscomisional La Salle, se efectuó la aplicación del cuestionario de detección de dificultades de aprendizaje (CEPA), que consta de 33 ítems con agrupaciones en 7 áreas, este es un instrumento adecuado para la evaluación individual en el aula ejecutada por el docente, mismo que permite evaluar los procesos cognitivos y del lenguaje (Pacheco, 2014).

Las dificultades de aprendizaje suelen ser un factor que interfiere de una manera significativa en el aprendizaje de los estudiantes. Con la indagación efectuada se ha logrado visualizar que un $20 \%$ de los estudiantes poseen problemas o déficit en ciertas áreas o asignaturas de la educación no asociados a la discapacidad, para efectuar el trabajo es importante realizar una investigación teórica profunda de las dificultades de diversos autores y web grafías, para conocer que son las dificultades de aprendizaje en estudiantes que no estén asociados a la discapacidad, qué factores son los que lo originan y las posibles implicaciones en el ámbito académico y personal del ser humano.

"Dentro del plano pedagógico al niño se le imposibilita seguir con éxito sus estudios en la enseñanza primaria, estos sujetos manifiestan problemas de habla, lectura, escritura y matemáticas. Tienen problemas de razonamiento, deletreo e interacción social" (Fiuza Asorey \& Fernández Fernández, 2014). Las dificultades de aprendizaje estarán presentes en los ámbitos educativos, y son los docentes los encargados de dar a conocer dichas observaciones, para ayudar a los niños con una intervención temprana.

El Instituto Nacional de Nivelación de Evaluación y Calidad de Sistema Educativo, con un estudio realizado en el año 2004, detectaron que el $16 \%$ de estudiantes de primaria han presentado un rendimiento bajo en el área de matemática y lengua castellana (INECSE, 2004). Con el análisis de los valores obtenidos en esta investigación los estudiantes suelen justamente presentar problemas en las materias básicas como la lectura, escritura y el cálculo, y para todo ello se requiere de una intervención desde que se está en los primeros años de educación primaria.

En lo que respecta al área de percepción de la información, un 9,10\% presentaba deficiencias. Los estudiantes hoy en día presentan falencias al momento de comprender la información y las instrucciones que da el docente dentro del aula de clase, estos factores pueden deberse a que, desde edades tempranas no han sido estimulados de la manera co- 
rrecta, mediante actividades de percepción auditiva, visual y táctil. Cuando una persona logra captar el mensaje que le transmite el emisor, puede desarrollar de mejor manera todas las tareas o actividades encomendadas. Los niños que no logran captar la información dada por el docente, tienen una memoria a corto plazo, es decir, olvidan con facilidad las instrucciones dadas. Por el contrario, la memoria a largo plazo es muy importante a la hora de realizar actividades diarias, este tipo de memoria da al cerebro la capacidad de almacenar información, acontecimientos y destrezas.

(González \& Muñoz, 2013), con sus estudios realizados agregaron que: "Las dificultades en el procesamiento de la información se da cuando el niño tiene problemas en las entradas y salidas, no se debe al esfuerzo ni al tipo de inteligencia, sino de cómo su cerebro reconoce y utiliza la información". El cerebro es el factor importante para el procesamiento de la información, por ello se debe estimular a los niños para que, día a día mejoren, y de esta manera empiecen a receptar y emitir información de manera correcta. Otro autor como Jiménez acotó que: Dentro de la escolarización se puede detectar que las habilidades visuales y del lenguaje, no han sido desarrolladas con satisfacción, y con ello ya traen dificultades en el aprendizaje dentro del aula. Los niños tienden fallar al receptar información. (Jiménez, 2004).

Durante la evaluación de cada una de las áreas investigadas se concuerda con lo que enfatiza, Jiménez, de manera que, la existencia de Dificultades de Aprendizaje no asociados a la discapacidad si se encuentra presente dentro de un aula de clase, los docentes junto a profesionales en educación pueden ayudar a corregir las falencias, para que los niños tengan un desarrollo normal y mayor comprensión en las áreas que están fallando. Si no se corrige desde la primera área que es la de recepción de infor- mación afectada hasta la de matemáticas, no se podrá ayudar en las demás. Este proceso se da en cadena, pues el desarrollo de cada uno ayuda a tener un aprendizaje significativo.

En lo que se refiere al área de expresión del lenguaje oral, los estudiantes casi en su totalidad, lo desarrollan de gran manera, con una buena capacidad de pronunciación y uso del lenguaje; el 1,8\% detectado debe de ser trabajado, y apoyar a que el niño pueda desarrollar y hacer un buen uso del lenguaje oral. Celdrán y Zamorano acotan que: "Las tendencias actuales sobre el desarrollo del lenguaje, es un camino integrador, tanto en aspectos psicológicos como orgánicos, formales como funcionales, ya que el desarrollo del lenguaje no puede estar separado de lo motriz, cognitivo y social" (Celdrán \& Zamorano, 2006).

En el área de atención - concentración - memoria, hubo la existencia de un $6 \%$ de niños que se encontraban entre lo deficiente y mal, a este factor se debe poner mucho cuidado, puesto que, de esta área depende el buen desarrollo del estudiante en su proceso de enseñanza aprendizaje. (Salomón, 2003) en lo referente al tema acota que: "La atención, concentración y memoria, son procesos que están estrechamente vinculados y que permiten que se pueda adaptar y desenvolver de manera eficaz en las diferentes situaciones que se les presenta diariamente". Con los resultados obtenidos y estudios realizados anteriormente por otros autores, se conoce que esta área necesita estar reforzada continuamente desde el inicio del proceso escolar, para que en un futuro no afecte en su rendimiento.

En lo que tiene que ver con los errores en la lectura, la población investigada casi en su totalidad muestra un buen dominio y desarrollo en la lectura. Solamente el 1\% de los niños tienen dificultad al leer, los escolares suelen por lo general titubear al leer, tiene una falta de co- 
nocimiento en las palabras o no comprende lo que lee. Este factor afecta en el desarrollo del aprendizaje, por la edad en que se encuentran deben saber decodificar con fluidez y tener la capacidad de retener cada uno de los contenidos aprendidos dentro del aula de clase. Los estudiantes con dificultades pueden tener una lectura disléxica o carencial, presentar fallas de ritmo y conocimiento.

Para despertar el interés y que los niños aprendan a leer, las condiciones en las que se encuentra deben ser adecuadas, es decir que los libros sean llamativos e interesantes, y que las relaciones con las demás personas o sus pares puedan ayudarlos. A nivel del sistema educativo, los docentes son los encargados de aplicar distintas formas de enseñanza, y no solamente un cambio de metodología. Esta ayuda estará ligada directamente a la superación de las deficiencias, observando e identificando (Galeano, 2013), para fortalecer los puntos débiles y apoyar en los puntos fuertes. En lo que respecta a la lectura este autor dice lo siguiente: "Los niños no se convierten en lectores gracias a las metodologías, sino que aprenden a leer cuando las condiciones con adecuadas" (Smith, 1999) citado por (UNESCO, 2016)

Dentro del área de la escritura, un 7,3\% de los estudiantes analizados han mostrado errores de escritura. Los niños tienden a tener problemas al momento de escribir, no saben cómo organizar y expresar todas sus ideas que están su mente. En el ámbito educativo al darse estas situaciones, los escolares pueden llegar a sentirse confundidos, y no encontrar la manera de corregir esta dificultad, esto se verá reflejado en que su escritura será ilegible, mezclarán diferentes tipos de letras, no respetaran los espacios, fallarán constantemente en su gramática y la ortografía, es decir presentaran dificultades para organizar toda la información al momento en el que van a escribir.
No hay que dejar de lado por más mínimo porcentaje de estudiantes que presenten dificultades en su lectura y escritura, por el contrario, centrar la atención en ellos aporta en la construcción de un buen proceso de enseñanza. Las investigaciones de Problemas de Aprendizaje corroboran este hecho, pues, a nivel nacional e internacional se dan estos casos y no hay que excluirnos, sino prestar más atención.

Las matemáticas, son otra área de gran relevancia en el ámbito educativo, pues en el desarrollo de la vida cotidiana está presente aquello. En la educación primaria los estudiantes muestran variedades de capacidades y diferentes ritmos de aprendizaje. El 12,7\% de niños observados tienen una gran dificultad en el desarrollo de las operaciones básicas, la suma, resta, multiplicación y división. El problema más común en estos cursos es la memorización de las tablas de multiplicar. En lo que tiene que ver con la suma y resta, pueden calcularla mediante el recuento y en la mayoría de los casos hacen uso de sus dedos para el conteo.

Todo esto se debe a que con las investigaciones realizadas han logrado demostrar que sí existen caminos para vencerla. El aprendizaje de las matemáticas, va ir de la mano la lectura y la escritura, pues estos aprendizajes son de vital importancia en la educación elemental y básica. Los problemas con las matemáticas hoy en día se han convertido en una preocupación de muchos docentes de aula o asignatura, especialmente, porque en su rendimiento se ven reflejadas las bajas calificaciones, y es un alto porcentaje de estudiantes que, en las instituciones educativas, bajan de calificaciones y terminan la escolaridad de forma obligatoria. La resolución de problemas es un eje importante en el proceso de enseñanza - aprendizaje, por ello es recomendable trabajarla en conjunto con las demás áreas de estudio.

En la investigación realizada el $20 \%$ de la po- 
blación muestra mayor inconveniente en la escritura y las matemáticas, dos áreas que son de mucha importancia para el desarrollo del proceso de enseñanza - aprendizaje. Con estudios ya realizados por otros autores entre ellos (Galindo, 2002), han dado a conocer que tanto en México como en otras ciudades internacionales, se ha tenido por lo menos de un 10\% a $15 \%$, de niños que han presentado este tipo de Dificultades, de la misma manera han determinado que, mientras más temprano se haga un diagnóstico, los estudiantes tendrán mejores posibilidades de recuperación. La detección temprana ayuda a superar en un gran porcentaje las dificultades de aprendizaje.

Dentro de la evaluación global de los aprendizajes, los docentes consideran que en el $10,91 \%$ de los estudiantes, si existe dificultad, de manera especial en las áreas de lengua o castellanoy matemáticas, puesto que, son dos ejes importancia en los aprendizajes, por ello siempre están en constante evaluación, al comenzar el año lectivo, durante y al final. Con la ayuda de aquello, pueden verificar en donde se encuentra más afectado el estudiante, y ver si su ritmo de aprendizaje es lento o normal, para poder llevarlos a una toma de decisiones y acompañar en el proceso educativo y de reforzamiento a aquellos que lo necesitan, sin dejar de lado a aquellos que tienen un rendimiento bueno. La evaluación ayuda a recoger la información necesaria del aprendizaje del niño, permitiendo no solo mejorar, sino también detectar dificultades.

Un buen proceso de enseñanza - aprendizaje se da cuando el docente busca las estrategias necesarias de llegar a sus estudiantes en los contenidos que imparte, trata de hacer la clase más llamativa para que pongan toda su concentración y a su vez tiene la capacidad de detectar y trabajar con aquellos que no alcanzan a seguir el ritmo de trabajo al igual que sus compañeros. A los estudiantes que tienen dificulta- des de aprendizaje se les debe incluir en todo momento, de no darse aquello ellos tienden a sentirse solos, afectando aún más al problema que tienen, y en muchas de las veces se da la deserción escolar que hoy en día preocupa a la educación nacional e internacional.

Con el cuestionario CEPA aplicado, los docentes consideran que el 100\% de los estudiantes, no tienen déficit en su inteligencia, ya que está dentro del parámetro de lo normal. Profesionales del campo educativo explican que existen niños que pueden tener una capacidad intelectual buena y al mismo tiempo pueden presentar dificultades en el aprendizaje. La dificultad de los estudiantes está en las áreas básicas de estudio.

Durante el proceso se enseñanza - aprendizaje, los docentes tienen la capacidad de observar a cada uno de los estudiantes, pero; no tienen un instrumento de detección de dificultades de aprendizaje no asociados a la discapacidad. Conforme ha avanzado la ciencia y la tecnología, existen una serie de instrumentos que puede utilizar dentro del aula, el inconveniente está en que no saben cómo usarlo. Por ello las instituciones educativas deben de asesorar y capacitar a los educadores, para que tengan conocimiento y puedan realizar la intervención pedagógica de las diferentes dificultades de aprendizaje existentes y que no están asociadas la discapacidad.

\section{Conclusiones}

Con los resultados obtenidos de la población total observada se identifica que un $20 \%$ de los estudiantes entre los dos cursos, muestran un resultado muy amplio de rasgos de Dificultades de Aprendizaje no asociados a la discapacidad, si no es tratado a tiempo en un futuro los estudiantes mostraran rasgos de dificultad más marcados, donde se volverá más difícil ayudarlos. Estos inconvenientes en el aprendizaje pueden ser debido a que, en los primeros 
años de escolarización los niños y niñas no desarrollaron de manera adecuada todas las áreas de estudio, y no fueron observados por los docentes de aula. Se infiere que aquellos docentes no centraron su atención en los niños que ya estaban presentando estos problemas, y se les promovió sin ninguna observación a cursos superiores.

Los niños de primaria tienden a fallar más en la comprensión de las operaciones y la comprensión de los enunciados. De esta manera los docentes deben agotar todos sus recursos para ayudar a los niños que lo necesitan. Los instrumentos de detección de dificultades de aprendizaje pueden ser utilizados para evaluar desde los primeros años de escolarización, sólo de esta manera se puede intervenir y dar seguimiento continuo. Las aportaciones de las nuevas tecnologías facilitan en la comunicación y el aprendizaje continuo que deben tener los estudiantes y los docentes, es decir estar a la par con la tecnología.

Las áreas más afectadas son las de escritura y matemáticas, puesto que, los estudiantes presentan desde los primeros años de escolarización han tenido vacíos, que conforme han pasado los años no han sido llenados. Los docentes deben conocer el nivel de competencia que presenta el estudiante, identificando de esta manera qué barreras de aprendizaje están presentes, con el fin de ayudarles a superar, y que vayan mejorando sus conocimientos.

\section{Referencias}

Andalucía. (2012). Dificultades de Aprendizaje de las Matemáticas. (R. d. enseñanza, Entrevistador)

Asociación Americana de Psiquiatría. (2014). Guía de cunsulta de los criterios diagnósticos del DSM-5. London: Asociación Americana de Psiquiatría.

Betancurt, L. G. (2010). Cuestionario de Rastreo de trastorno de aprendizaje (CEPA) en niños de edad escolar.

Bravo Valdivieso, L. (1979). CUESTIONARIO DE EVALUACIÓN DE PROBLEMAS DE APRENDIZAJE (CEPA). Universidad Austral.

Celdrán, M., \& Zamorano, F. (2006). TRASTORNOS DE LA COMUNICACIÓN Y EL LENGUAJE. Murcia.

DSM-V. (2013). Manual Diagnóstico y Estadístico de los trastornos mentales. (A. E. Psiquiatría, Entrevistador)

Fiuza Asorey, M. J., \& Fernández Fernández, M. P. (2014). Dificultades de Aprendizaje y Trastornos del Desarrollo. Madrid: Ediciones Pirámide.

Galeano Sánchez, J. C. (2013). El fenómeno del consumo, entendido desde su historicidad y su impacto social. Ad-Gnosis, 2(2), 129-146.

Galindo, D. (2002). Detección Temprana de los Problemas de Aprendizaje. Madrid.

García, N.J. (2010). Manual de Dificultades de Aprendizaje, Lenguaje, Lecto-Escritura y Matemáticas. Madrid España: Narcea.

González, B., \& Muñoz, E. (2013). ESTIMULACIÓN COGNITIVA Y TECNOLOGIAS DE LA INFORMACIÓN Y LA COMUNICACIÓN. Barcelona: FOUC, Fundación para la Universidad Obera de Catalunya.

INECSE. (2004). Instituto Nacional de Evaluación y Calidad de Sistema Educativo. Datos de evaluación y calidad de la educación del Ministerio de Educación y Ciencia.

Jiménez, A. A. (2004). Introducción a las dificultades de aprendizaje. Madrid: Interamericana de España.

Pacheco, B. (2014). Desarrollo cognitivo y adquisición solfistica en niños de ocho a doce años. Pensamiento Americano, 7(12), 11-19.

Romero Pérez, J., \& Cerván, R. (2005). Dificultades en el 
Aprendizaje, Unificación de Criterios Diagnóstico. Málaga.

Salomón, M. (2003). Atención y Memoria: su influencia en el aprendizaje. Curso de Capacitación Docente en Neurociencias, 6.

UNESCO. (2016). Aportes para la enseñanza de la lectura. (l. C. Organización de las Naciones Unidas para la Educación, Entrevistador)

Vega. (2005). Recursos metodológicos aplicados al trabajo con niños que presentan dificultades de aprendizaje. Lima - Perú: CESIP.

2018, Vol. 11(21) 169-180 @ The Author(s) 2018

Reprints and permission: www.americana.edu.co

https://www.coruniamericana.edu.co/publicaciones/ojs/index.php/pensamientoamericano/index 